\title{
Validade de conteúdo da Avaliação para Melhoria da Qualidade da Estratégia Saúde da Família
}

\author{
I ${ }^{1}$ Thiago Dias Sarti, ${ }^{2}$ Ethel Leonor Noia Maciel, ${ }^{3}$ Carlos Eduardo Aguilera Campos, \\ ${ }^{4}$ Eliana Zandonade, ${ }^{5}$ Gustavo Enrico Cabral Ruschi I
}

Resumo: O objetivo do trabalho é analisar a validade de conteúdo dos instrumentos de autoavaliação que compõem a Avaliação para Melhoria da Qualidade da Estratégia Saúde da Família (AMQ). Trata-se de um estudo ecológico, retrospectivo, de análise de dados secundários, com utilização dos dados disponíveis de 46 dos 64 municípios que aderiram ao processo de AMQ no Espírito Santo até Dezembro de 2007, totalizando 241 equipes de saúde da família. Foram calculadas as médias de percentual de respostas "sim" que as equipes de saúde davam em cada padrão de qualidade do AMQ. Encontrou-se uma não linearidade entre a complexidade de implementação dos processos de trabalho sugeridos pelo AMQ a partir do elenco de padrões em cada estágio de qualidade e a autoavaliação das equipes de saúde da família dos municípios estudados. A identificação da não linearidade da relação entre os estágios de qualidade do AMQ e a complexidade de implantação dos processos de trabalho preconizados pelos padrôes de qualidade evidencia a necessidade de se reorganizar os cadernos de autoavaliação, de forma a encontrar uma correlação mais consistente entre dificuldade de implantação dos processos de trabalho no cotidiano da ESF e estágios de qualidade.

> Palavras-chave: atenção primária à saúde, Saúde da Família, qualidade da assistência à saúde, Avaliação para Melhoria da Qualidade da Estratégia Saúde da Família (AMQ).

\author{
${ }^{1}$ Médico de Família e \\ Comunidade. Mestre em Saúde \\ Coletiva pela Universidade \\ Federal do Espírito Santo \\ (UFES). Professor Assistente \\ do Departamento de Medicina \\ Social, Centro Biomédico, \\ UFES. Endereço eletrônico: \\ thiagosarti@yahoo.com.br \\ 2 Doutora em Epidemiologia \\ pelo IMS-UERJ; Professora \\ Adjunta do Departamento \\ de Enfermagem, Centro \\ Biomédico, UFES. Endereço \\ eletrônico: emaciel@ndi.ufes.br \\ ${ }^{3}$ Doutor em Medicina \\ Preventiva pela USP; Professor \\ Adjunto do Programa de \\ Atenção Primária à Saúde, \\ Faculdade de Medicina, \\ Universidade Federal do Rio \\ de Janeiro (UFRJ). Endereço \\ eletrônico: cadu@vetor.com.br \\ ${ }^{4}$ Doutora em Estatística pela \\ USP. Professora Adjunta do \\ Departamento de Estatística, \\ Centro de Ciências Exatas, \\ UFES. Endereço eletrônico: \\ elianazandonade@uol.com.br \\ ${ }^{5}$ Mestre em Obstetrícia pela \\ UNIFESP. Professor Assistente \\ do Programa de Interação \\ Serviço, Ensino e Comunidade, \\ Centro Universitário Vila Velha \\ (UVV). Endereço eletrônico: \\ gruschi@terra.com.br
}

Recebido em: 13/05/2010 Aprovado em: 21/04/2011 


\section{Introdução}

O Programa Saúde da Família (PSF) foi criado e implementado no ano de 1993 e 1994, respectivamente. A partir de 1995, iniciam-se a fusão PACS/PSF e um processo de implantação do programa em todo o território nacional, o que ocorre principalmente a partir de 1997, tratando-se agora de um modelo de reorientação tecnoassistencial do sistema de saúde brasileiro denominado Estratégia de Saúde da Família - ESF (BRASIL, 1997).

Novas práticas são preconizadas, como o trabalho em equipe interdisciplinar, a ênfase na promoção e prevenção de saúde, o vínculo e a responsabilização com a população, a adscrição de clientela e a vigilância da saúde. Os princípios organizacionais e operacionais são definidos a partir de contratos entre o nível federal e municipal, e incluem: caráter substitutivo com relação à rede de atenção primária tradicional; territorialização; processos de trabalho direcionados aos principais problemas de saúde da população; planejamento local compartilhado com a comunidade adscrita e seus movimentos organizados; contextualização familiar, cultural e comunitária do processo saúde-doença; e fomento da cidadania (BRASIL, 2006, 1997).

Vários avanços foram obtidos com a ESF, dentre eles: maior acesso aos cuidados básicos de saúde, principalmente por parte da população em piores condiçōes socioeconômicas (PAIVA; BERSUSA, ESCUDER, 2006); uma redução significativa da mortalidade infantil nas áreas cobertas pela estratégia (MACINKO et al., 2007; MACINKO; GUAINAIS; SOUZA, 2006); melhor controle de doenças crônicas como hipertensão e diabetes mellitus (ARAUJO; GUIMARÃES, 2007); e resultados positivos em termos de internação hospitalar devido a condições sensíveis à Atenção Primária à Saúde (APS) (GUAINAIS; MACINKO, 2009; ABREU; CESAR; FRANÇA, 2007). No entanto, ainda se enfrentam problemas com relação ao financiamento da APS no país e à infraestrutura das unidades de saúde da família, tais como equipes de saúde pouco qualificadas para o trabalho em APS, alta rotatividade dos profissionais dessas equipes, vínculos empregatícios precários em grande parte dos municípios do país, gestão ineficiente para o contexto da ESF, deficiências com a atenção integral à saúde da população e entraves na implantação de ações preventivas e de educação em saúde (CONILL, 2008). 
Com esse panorama, a discussão em torno da qualidade dos serviços primários de saúde se impõe para a consolidação dos princípios do Sistema Único de Saúde (SUS) (CAMPOS, 2005). Nesse sentido, com o intuito de institucionalizar a avaliação na APS e qualificá-la, é lançada, dentre outras estratégias, a Política Nacional de Avaliação da Atenção Básica em Saúde, no ano de 2003, pela Coordenação de Atenção Básica do Ministério da Saúde, sendo que a Avaliação para Melhoria da Qualidade em Atenção Primária (AMQ) é um projeto estratégico que integra essa política (BRASIL, 2005b). O AMQ tem por objetivos: desenvolver metodologia de avaliação para a melhoria contínua da qualidade da ESF; definir padrões de qualidade para esta; construir instrumento de monitoramento e avaliação; desenvolver estratégias de implantação da ESF de forma qualificada no nível local; contribuir com o desenvolvimento da capacidade técnica em monitoramento e avaliação nas Secretarias Estaduais de Saúde e municípios brasileiros; e induzir boas práticas em atenção primária (BRASIL, 2005a, 2005b).

O momento instrumental da AMQ consiste na aplicação de instrumentos de autoavaliação pelos atores da APS - sejam estes gestores, coordenadores da APS, de unidades de saúde ou equipes de saúde da família - com o objetivo de instituir um processo de autoavaliação da qualidade da ESF do município em seus diversos componentes, municiando o ator em seu processo decisório para a melhoria da qualidade da APS. Esses instrumentos de auto-avaliação - cinco cadernos no total - são compostos por 300 padrões de qualidade que versam sobre elementos de estrutura, processos e resultados, todos considerados essenciais para uma efetiva implementação da ESF, sendo que a elaboração desses instrumentos partiu de premissas básicas como livre adesão com utilização facultativa dos instrumentos pelo município e ausência de mecanismos que levassem a penas ou recompensas por eventuais resultados alcançados (BRASIL, 2005b).

Contudo, embora seja uma política oficial de melhoria da qualidade da APS no Brasil, ainda são poucos os trabalhos que realizaram estudos com avaliaçôes sobre os aspectos metodológicos e práticos do AMQ. Uma das questôes que se colocam na análise dessa metodologia é a consistência interna dos instrumentos quanto à distribuição dos padrões de qualidade entre os cinco estágios de qualidade proposto no arcabouço teórico do AMQ ("elementar", "em desenvolvimento", "consolidado", "bom”, “avançado"). Cada padrão de 
qualidade do AMQ é enquadrado em um determinado estágio de qualidade, pressupondo, ao menos em tese, graus de dificuldade crescentes para o seu cumprimento (Figura 1). A ideia central é a de que gestores e profissionais de saúde alcancem níveis crescentes de conformidade com os padrões de qualidade do AMQ, obedecendo, em certa medida, aos estágios incrementais de qualidade expostos acima (BRASIL, 2005b).

Este trabalho busca avaliar se os padrōes de qualidade do AMQ apresentam dificuldades crescentes de implantação pelos profissionais da Saúde da Família, na mesma medida e nas mesmas proporções em que foram atribuídos pelos seus idealizadores, com a adoção dos cinco estágios de qualidade. Partese da hipótese de que as médias de conformidade dos padrōes de qualidade dos estágios mais avançados ( $\mathrm{B}$ e $\mathrm{A}$ ) devem ser progressivamente menores que as de padrões pertencentes a estágios mais elementares (E, D, C). Portanto, este trabalho tem como objetivo testar essa hipótese e analisar a validade de conteúdo dos instrumentos de autoavaliação que compõem o AMQ no que diz respeito à adequação dos cinco estágios de qualidade de crescente complexidade de implementação, como forma de subsidiar o processo de melhoria da qualidade da ESF municipal.

Para se alcançar tais objetivos, optou-se por estudar a dimensão "Consolidação do Modelo de Atenção" e suas respectivas subdimensões: "Organização do Trabalho"; "Acolhimento, Humanização e Responsabilização"; "Promoção da Saúde"; "Participação Comunitária" e "Vigilância à Saúde I: Ações Gerais". Os padrōes de qualidade pertencentes a essas subdimensões são respondidos pelas equipes de saúde da família, incluindo os profissionais de nível médio, técnico e superior. Essa escolha se deu pela relevância dos temas aí tratados para a consolidação da ESF no Brasil, e também pela amplitude dos profissionais envolvidos na resposta a estes padrões.

\section{Metodologia}

Realizou-se estudo ecológico, retrospectivo, de análise de dados secundários. Foram utilizados os dados disponíveis de 46 dos 64 municípios que aderiram ao processo de Avaliação para Melhoria da Qualidade da Estratégia de Saúde da 
Família (AMQ) no Espírito Santo até Dezembro de 2007. Esse limite de tempo

refere-se ao primeiro momento avaliativo do AMQ realizado pelos municípios estudados, tal como preconizado pelo Ministério da Saúde (MS).

A escolha do estado do Espírito Santo se deve ao alcance da implementação do AMQ nesse estado, em virtude de uma orientação da gestão estadual em considerar o AMQ como peça salutar para a organização dos sistemas de saúde municipais. Isso permitiu que a amostra de municípios fosse relevante e significativa, garantindo a consistência dos resultados aqui apresentados. Em termos de universo de equipes de saúde da família respondentes, a amostra de 46 municípios corresponde a 241 equipes ou $48 \%$ das equipes implantadas em Dezembro de 2007 no ES.

Figura 1 - Estágios de Qualidade da Avaliação para a Melhoria da Qualidade da Estratégia Saúde da Família (AMQ)*

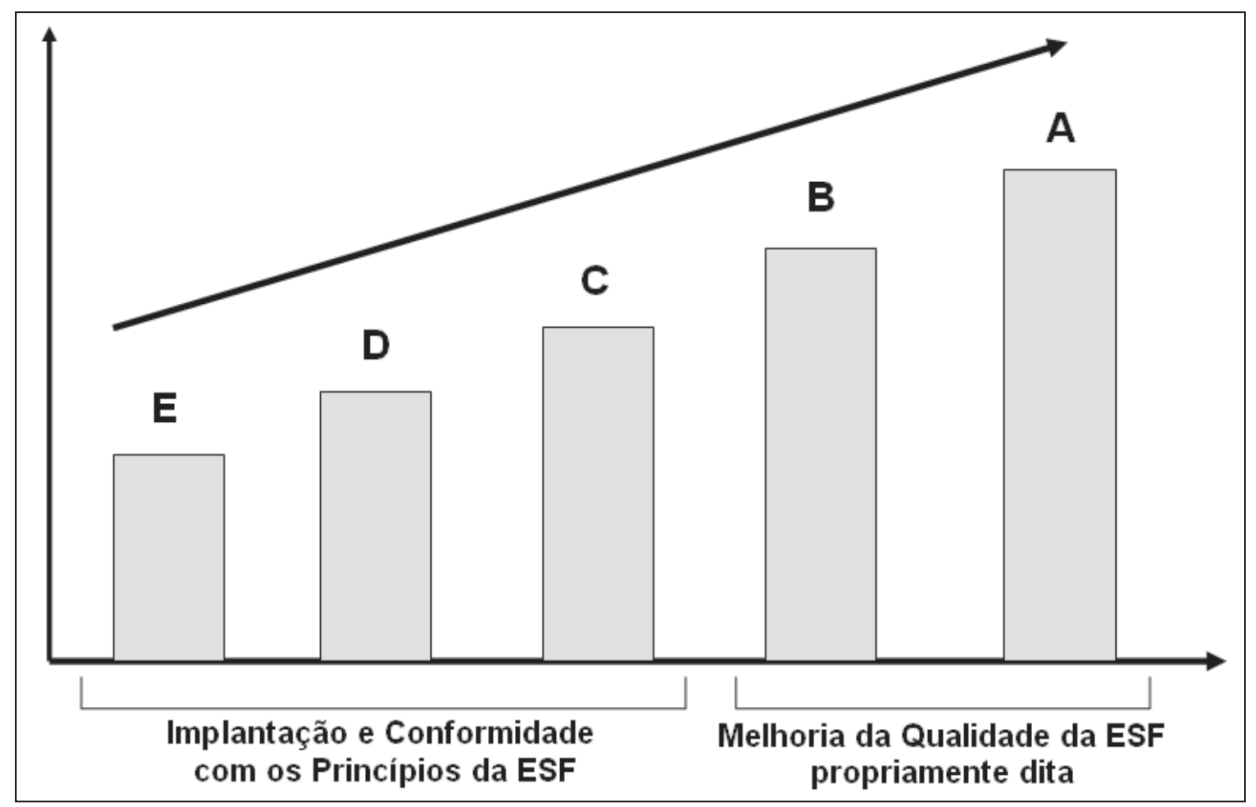

Fonte: Adaptado de Departamento de Atenção Básica / Ministério da Saúde (BRASIL, 2005b) ESF: Estratégia Saúde da Família

*A - Avançado; B - Bom; C - Consolidado; D - Desenvolvimento; E - Elementar 
O banco de dados do AMQ foi disponibilizado pela Coordenação de Acompanhamento e Avaliação do Departamento de Atenção Básica do Ministério da Saúde (CAA / DAB / MS), em formato compatível com o programa Office Excel ${ }^{\circledR}\left(\right.$ Microsoft $\left.^{\circledR}\right)$. Esse banco contém os dados referentes às respostas aos cinco cadernos de avaliação do AMQ de 46 dos 64 municípios que aderiram ao instrumento.

Os padrōes de qualidade do AMQ são variáveis categóricas nominais dicotômicas, configurando-se em respostas do tipo "sim", caso o padrão seja executado pela equipe de saúde, e "não", caso não haja implantação em conformidade com o preconizado (BRASIL, 2005b). A unidade de análise considerada foram as equipes agrupadas por município e, dessa forma, procedeuse o cálculo da média de percentual de respostas "sim" em cada padrão para cada município. Também se procedeu à análise do desvio padrão de cada variável estudada, permitindo aferiçôes quanto à variabilidade nos processos das diferentes equipes dos municípios participantes. Para análise do banco de dados, utilizou-se o pacote estatístico SPSS 15.0 (SPSS Inc., 2006) após reorganização das planilhas a partir do banco original de dados.

Como os instrumentos de autoavaliação do AMQ dispõem os padrões de qualidade de acordo com subdimensôes estabelecidas a partir de eixos analíticos dos processos de trabalho da ESF, optou-se por executar a análise dos dados respeitando essa disposição, agrupando-se os padrões nas cinco subdimensões referentes à dimensão "Consolidação do Modelo de Atenção" do componente "Equipe" do AMQ. No total, o Caderno 4 é composto por 70 padrões de qualidade divididos nas seguintes subdimensões: "Organização do Trabalho em Saúde da Família" (21 padrões); "Acolhimento, Humanização e Responsabilização" (12); "Promoção da Saúde" (21); "Participação Comunitária e Controle Social" (4); e "Vigilância à Saúde: Ações Gerais da Estratégia Saúde da Família" (12).

As médias foram analisadas com base nos pontos de corte previamente definidos pelos autores, considerando um padrão de resposta esperado, isto é, foram analisadas como um nível mínimo compatível com uma lógica incremental de qualidade, com percentuais de respostas positivas progressivas ao cumprimento de padrões de acordo com estágios de qualidade E, D, C, B, A (tabela 1). 

para a Melhoria da Qualidade da Estratégia Saúde da Família (AMQ) Espírito Santo, 2009

\begin{tabular}{l|c}
\hline $\begin{array}{c}\text { Estágio de } \\
\text { Qualidade }\end{array}$ & $\begin{array}{c}\text { Mínimo exigido em média de percentual de respostas positivas } \\
\text { aos padróes conforme sua implantação no processo de trabalho } \\
\text { das equipes de saúde da família }\end{array}$ \\
\hline Elementar & $80 \%$ ou mais \\
\hline Desenvolvimento & $60 \%-79,9 \%$ \\
\hline Consolidado & $40 \%-59,9 \%$ \\
\hline Bom & $20 \%-39,9 \%$ \\
\hline Avançado & $10 \%-19,9 \%$ \\
\hline
\end{tabular}

Espera-se que as médias de respostas "Sim" aos padrões de qualidade do AMQ sigam uma tendência decrescente conforme se avança nos estágios de qualidade, sendo o estágio "elementar" em tese o que apresentaria maiores médias e o estágio "avançado", as menores. Além de ser parâmetro para avaliação da qualidade autorreferida da ESF, essa análise permite avaliar a consistência do instrumento AMQ quanto à distribuição dos padrões de qualidade em seus diversos estágios.

Foram consideradas todas as diretrizes e normas regulamentadoras de pesquisas envolvendo seres humanos previstas na Declaração de Helsinque e na resolução 196/96 da Comissão Nacional de Ética em Pesquisa (CONEP), com aprovação pelo Comitê de Ética em Pesquisa (CEP) do Centro de Ciências da Saúde da Universidade Federal do Espírito Santo, em 26 de Junho de 2008 registro no CEP n ${ }^{\circ}$ 035/08. Os dados foram cedidos pelo Ministério da Saúde (MS), a partir da solicitação oficial pelo Programa de Pós-Graduação em Saúde Coletiva da Universidade Federal do Espírito Santo (PPGSC/UFES), ao Departamento de Atenção Básica do MS. Uma das exigências protocolares do AMQ é a manutenção do sigilo quanto à identidade dos municípios e equipes participantes do processo de avaliação. Esse requisito foi seguido irrestritamente no desenvolvimento desta pesquisa. 


\section{Resultados}

As médias de percentual de respostas "sim" aos padrões de qualidade do AMQ em cada subdimensão estão descritas na tabela 2, distribuídas conforme os cinco estágios aos quais pertencem cada padrão de qualidade. A descrição da correspondência das médias de respostas "sim" com padrões de qualidade do AMQ e seus respectivos estágios incrementais pode ser vista na tabela 3.

Tabela 2 - Média de respostas "sim" aos padrões de qualidade da Avaliação para a Melhoria da Qualidade da Estratégia Saúde da Família (AMQ) Espírito Santo, 2009

\begin{tabular}{|c|c|c|c|c|c|c|c|c|c|c|}
\hline \multirow[b]{3}{*}{ Subdimensóes } & \multicolumn{10}{|c|}{ Estágios de Qualidade* } \\
\hline & \multicolumn{2}{|c|}{$\mathbf{E}$} & \multicolumn{2}{|c|}{$\mathbf{D}$} & \multicolumn{2}{|c|}{$\mathrm{C}$} & \multicolumn{2}{|c|}{ B } & \multicolumn{2}{|c|}{ A } \\
\hline & $\mathbf{m}$ & $d p$ & m & $d p$ & $\mathbf{m}$ & $d p$ & m & $\mathrm{dp}$ & $\mathbf{m}$ & dp \\
\hline $\begin{array}{l}\text { Organização do } \\
\text { Trabalho }\end{array}$ & 62,4 & 25,4 & 62,4 & 15,9 & 47 & 21 & 27,7 & 5,9 & 15,1 & 4,7 \\
\hline $\begin{array}{l}\text { Acolhimento, } \\
\text { Humanização }\end{array}$ & 85,00 & 5,2 & 47,2 & 31,3 & 40,1 & 29,7 & 41 & 6,6 & 68,7 & 7,1 \\
\hline Promoção da Saúde & 66,60 & 33 & 40,2 & 25,9 & 26,9 & 14,3 & 14,7 & 8,9 & 6,1 & 3 \\
\hline $\begin{array}{l}\text { Participação } \\
\text { Comunitária } \dagger\end{array}$ & - & - & 7,2 & 17,7 & 7,1 & 18,8 & 24,3 & 28,1 & 8,5 & 18 \\
\hline $\begin{array}{l}\text { Vigilância Saúde I: } \\
\text { Açōes Gerais ESF }\end{array}$ & 79,80 & 4,2 & 65,1 & 34 & 81,3 & 9,7 & 49,9 & 5,7 & 30,6 & 18,2 \\
\hline
\end{tabular}

* Estágios de Qualidade dos padrões de qualidade do AMQ: E - Elementar; D - Desenvolvimento;

C - Consolidado; B - Bom; A - Avançado.

m: média; dp: desvio padrão;

† Não há padrōes de qualidade no estágio elementar de qualidade na subdimensão Participação Comunitária 
Tabela 3 - Análise da tendência das médias de percentual de respostas "sim" aos padróes incrementais de qualidade da Avaliação para a Melhoria da Qualidade da Estratégia Saúde da Família (AMQ) por Estágio de Qualidade-Espírito Santo, 2007

\begin{tabular}{l|l}
\hline \multicolumn{1}{c|}{ Subdimensão } & \multicolumn{1}{c}{ Tendência* } \\
\hline $\begin{array}{l}\text { Organização do } \\
\text { Trabalho }\end{array}$ & $\begin{array}{l}\text { As médias de respostas "sim" aos padrões dos estágios E e D de } \\
\text { qualidade são semelhantes, com um nível decrescente de respostas } \\
\text { "sim" a partir do estágio C de qualidade; }\end{array}$ \\
\hline $\begin{array}{l}\text { Acolhimento e } \\
\text { Humanização }\end{array}$ & $\begin{array}{l}\text { Apresenta uma tendência decrescente nas médias de respostas "sim" aos } \\
\text { padróes dos estágios de qualidade de E até C, com média semelhante } \\
\text { de C e B, sendo o último estágio de qualidade (A) com valores muito } \\
\text { acima do esperado (10-20\%); }\end{array}$ \\
\hline Promoção da Saúde & $\begin{array}{l}\text { Apresenta tendência decrescente nas médias de respostas "sim" aos } \\
\text { padróes do estágio E ao A de qualidade; }\end{array}$ \\
\hline $\begin{array}{l}\text { Participação } \\
\text { Comunitária }\end{array}$ & $\begin{array}{l}\text { Apresenta níveis semelhantes nas médias de respostas "sim" aos padrões } \\
\text { dos estágios D, C e A de qualidade, sendo que a média do estágio B } \\
\text { supera os demais e atinge o nível esperado (20\%); }\end{array}$ \\
\hline $\begin{array}{l}\text { Vigilância à Saúde I: } \\
\text { Açóes Gerais da ESF }\end{array}$ & $\begin{array}{l}\text { Apresenta tendência decrescente nos níveis das médias de respostas } \\
\text { "sim" aos padrōes dos estágios E a A, exceto em C cuja média supera a } \\
\text { dos demais estágios de qualidade; }\end{array}$ \\
\hline
\end{tabular}

*Estágios de Qualidade dos padrōes de qualidade do AMQ: E-Elementar; D - Desenvolvimento; C - Consolidado; B - Bom; A - Avançado.

No geral, a única subdimensão na qual as médias de percentual de respostas "sim" aos padrões de qualidade seguem uma tendência decrescente e linear com o aumento de complexidade dos estágios de qualidade é a referente à promoção da saúde. Em todas as outras subdimensões, pode-se notar alguma discrepância em determinados estágios de qualidade que os coloquem ou acima dos precedentes mais elementares ou abaixo dos estágios, ao menos em tese, mais complexos.

Esses resultados sugerem uma não linearidade entre a complexidade de implementação dos processos de trabalho sugeridos pelo $\mathrm{AMQ}$, a partir do elenco de padrões de qualidade em cada estágio, e a autoavaliação das equipes de saúde da família dos municípios estudados quanto a seus processos de trabalho. A média de respostas "sim" aos padrões de qualidade, muito acima da esperada, para o estágio avançado de qualidade na subdimensão "Acolhimento, Humanização e Responsabilização" exemplifica esse achado. 
Observaram-se, particularmente, médias abaixo do esperado na maioria dos estágios de qualidade das subdimensōes "Promoção da Saúde" e "Participação Comunitária e Controle Social". Contudo, médias dentro do esperado são encontradas em vários estágios de qualidade das subdimensões "Organização do Trabalho em Saúde da Família", "Acolhimento, Humanização e Responsabilização" e "Vigilância à Saúde: Ações Gerais da Estratégia Saúde da Família".

\section{Discussão}

As ações para a melhoria da qualidade da APS no Brasil são fundamentais para a consolidação do SUS como política social que efetivamente garanta o direito à saúde de todos os cidadãos brasileiros (CAMPOS, 2005), o que atesta a importância de uma política nacional de melhoria da qualidade da ESF, como éo caso do AMQ. Nesse sentido, trabalhos que contribuam com o aperfeiçoamento dos instrumentos que compõem esta estratégia são importantes por se tratar de política recente e ainda pouco difundida no universo de municípios do país, embora seja considerada pelo Ministério da Saúde como eixo fundamental na reorientação do modelo brasileiro de atenção primária. Além disso, instrumentos desse tipo, utilizados em diversas experiências internacionais de monitoramento e melhoria da qualidade da APS, costumam sofrer revisóes e reedições periódicas para a sua reutilização conforme o tempo (RACGP, 2000).

Os municípios optam livremente pela adesão à proposta, podendo utilizar os instrumentos do AMQ como subsídio de orientação na gestão da APS. O resultado da aplicação do AMQ é um diagnóstico de situação da organização e funcionamento dos serviços e suas práticas, tendo por base as diretrizes oficiais que norteiam a organização da ESF. Devido a seu caráter prático e pedagógico, o AMQ possibilita a elaboração de planos de ação para a melhoria da qualidade da ESF, de forma contínua e participativa, subsidiando os atores municipais na gestão do sistema de saúde (BRASIL, 2005b).

A identificação da não linearidade da relação entre os estágios de qualidade do AMQ e a complexidade de implantação dos processos de trabalho preconizados pelos padróes de qualidade evidencia a necessidade de se reorganizar os cadernos de autoavaliação, estabelecendo uma nova ordenação dos padrões de qualidade em seus respectivos estágios, de forma a se encontrar uma correlação mais consistente entre dificuldade de implantação dos processos de trabalho no cotidiano da ESF 
e estágios de qualidade. De outra forma, uma maior reflexão deve ser feita quanto à pertinência desses estágios de qualidade dentro do $\mathrm{AMQ}$, cabendo questionar se essa disposição teórica dos padrōes de qualidade efetivamente traz maior racionalidade à implantação do AMQ nos municípios. Isso também se torna relevante na medida em que pode aflorar aspectos metodológicos importantes que influenciarão a formulação de outros instrumentos de melhoria da qualidade.

Venâncio et al. (2008), em estudo realizado em São Paulo, mostraram que os atores envolvidos com a implantação do AMQ nos municípios paulistas queixavam-se das dificuldades de compreensão do que se queria com determinados padrões de qualidade em termos de processos de trabalho, o que implicava em certa resistência e desânimo na aplicação dos cadernos de autoavaliação. Isto traz à tona a importância de tornar esses cadernos simples e consistentes o suficiente para que todos os atores envolvidos, que pertencem a distintas categorias profissionais e possuem formação acadêmica heterogênea, compreendam e se sintam pertencentes a essa estratégia de melhoria da qualidade, o que vai estar diretamente relacionado com os resultados que se alcançarão com o AMQ (GEBOERS, 2001). Do contrário, como entender que uma equipe de saúde tenha um desempenho muito melhor no estágio avançado de qualidade que no estágio "em desenvolvimento", se o que se espera é que a complexidade seja crescente?

Outro ponto de relevância é o estabelecimento de critérios para análise dos consolidados de respostas das equipes de saúde pela gestão municipal, estadual e federal (BLACK; GRUEN, 2005). Parâmetros de avaliação não estão disponíveis na literatura e aqui se faz uma proposta para tal. Afirma-se que o AMQ pode funcionar como uma ferramenta de gestão para esses atores, ao identificar, com maior riqueza de detalhes, o cotidiano de gestores e profissionais de equipes de saúde. Por outro lado, os próprios profissionais das equipes de saúde podem se beneficiar de parâmetros de análise de sua realidade prática, contribuindo para um juízo de valor mais consistente e uma melhor tomada de decisão na melhoria da qualidade dos processos de trabalho (BODSTEIN, 2009).

Por último, salientam-se os limites observados na estruturação da ESF no ES, em particular no que concerne à promoção da saúde e à participação popular. Pode-se considerar esses campos de saberes e práticas como dois dos pilares da mudança de modelo de atenção almejado com a implantação do SUS, no qual se busca a integralidade da atenção e a orientação das práticas sanitárias em acordo com as necessidades de saúde da população, além de uma contextualização do 
processo saúde-doença no lócus de interação de seus determinantes fundamentais, dentre eles os de ordem social, política, econômica e cultural (BRASIL, 2006, 2001). Outros trabalhos já demonstraram as fragilidades dessas duas esferas na ESF (ROCHA et al., 2008; ALVES; NUNES, 2006), sendo que este estudo fortalece a compreensão dos entraves da APS no Brasil, exigindo esforços no sentido de superá-los ao se entender que a promoção da saúde e a participação popular são eixos fundamentais na melhoria da atenção à saúde da população.

Alguns limites deste estudo se originam das características de seu próprio desenho metodológico, sendo que os resultados de estudos ecológicos devem ser considerados com a devida cautela em locais distintos daqueles onde foram produzidos (ROTHMAN; GREENLAND; LASH, 2008). Além disso, o fato do AMQ ser uma metodologia de autoavaliação da qualidade, aqui se entendendo qualidade como o grau de conformidade com certos padróes, levanta a questão de em que medida é possível afirmar categoricamente que certos processos, indicados como sendo feitos, são de fato praticados pelas equipes de saúde, o que remete também à concepção de avaliação dos participantes. Contudo, se uma autoavaliação excessivamente positiva dos processos de trabalho e uma percepção de avaliação por um viés punitivo predominassem entre os profissionais, seria razoável esperar um resultado, em termos de média de respostas "sim", muito melhor do que o encontrado.

Dessa forma, espera-se que os resultados aqui apresentados possam contribuir para um aperfeiçoamento dos instrumentos do AMQ e para uma tomada de decisão mais consistente dentro de processos de melhoria contínua da qualidade no âmbito da APS, o que se constitui como práxis indispensável para o sistema de saúde público brasileiro.

\section{Referências}

ABREU, D.M.X.; CÉSAR, C.C.; FRANÇA, E.B. Relação entre as causas de morte evitáveis por atenção à saúde e a implementação do Sistema Único de Saúde no Brasil. Rev Panam Salud Publica, v. 21, n. 5, p. 282-91, 2007.

ALVES, V.S.; NUNES, M.O. Educação em Saúde na atenção médica ao paciente com hipertensão arterial no Programa Saúde da Família. Interface, Botucatu, v. 10, n. 19, p. 131-47, 2006.

ARAUJO, J.C.; GUIMARÃES, A.C. Controle da hipertensão arterial em uma unidade de saúde da família. Rev Saude Publica, v. 41, n. 3, p. 368-74, 2007.

BLACK, N.; GRUEN, R.. Understanding Health Services. Berkshire: Open University Press, 2005. 
BODSTEIN, R. Processo decisório e avaliação em saúde: ampliando o debate sobre o

Programa Saúde da Família. Cien Saude Colet, v. 14, n. supl. 1, p. 1336-45, 2009.

BRASIL. Ministério da Saúde. Avaliação da Atenção Básica em Saúde: caminhos da institucionalização. Brasília: Ministério da Saúde, 2005a.

. Avaliação para Melhoria da Qualidade Qualificação da Estratégia Saúde da Família. Brasília: Ministério da Saúde, 2005b.

. Política Nacional de Atenção Básica. Brasília: Ministério da Saúde, 2006.

. Programa Saúde da Família. Brasília: Ministério da Saúde, 2001.

- Saúde da Família: uma estratégia para reorientação do modelo assistencial. Brasília: Ministério da Saúde, 1997.

CAMPOS, C.E.A. Estratégias de avaliação e melhoria contínua da qualidade no contexto da Atenção Primária à Saúde. Rev Bras Saúde Mater Infant, v. 5, n. suppl. 1, p. s63-s69, 2005.

CONILL, E.M. Ensaio histórico-conceitual sobre a Atenção Primária à Saúde: desafios para a organização de serviços básicos e da Estratégia Saúde da Família em centros urbanos no Brasil. Cad Saúde Publica, v. 24, n. supl. 1, p. s7-s27, 2008.

GEBOERS, H. et al. Continuous quality improvement in small general medical practices: the attitudes of general practitioners and other practice staff. Int J Qual Health Care, v. 13, n. 5, p. 391-7, 2001.

GUANAIS, F.C.; MACINKO, J. The health effects of primary care decentralization in Brazil. Health Aff, v. 28, n. 4, p. 1127-35, 2009.

MACINKO, J.; GUANAIS, F.C.; SOUZA, M.F.M. Evaluation of the impact of the Family Health Program on infant mortality in Brazil, 1990-2002. J Epidemiol Community Health, v. 60, p. 13-19, 2006.

MACINKO, J. et al. Going to scale with community-based primary care: An analysis of the family health program and infant mortality in Brazil, 1999-2004. Social Science \& Medicine, v. 65, n. 10, p. 2070-80, 2007.

PAIVA, D.C.P.; BERSUSA, A.A.S.; ESCUDER, M.M.L. Avaliação da assistência ao paciente com diabetes e/ou hipertensão pelo Programa Saúde da Família do Município de Francisco Morato, São Paulo, Brasil. Cad Saude Publica, v. 22, n. 2, p. 377-85, 2006.

THE ROYAL AUSTRALIAN College of General Practitioners. Standards for General Practices. Melbourne, 2000.

ROCHA, P. M. et al. Avaliação do Programa Saúde da Família em municípios do nordeste brasileiro. Cad Saúde Publica, v. 24, n. supl. 1, p. s69-s78, 2008.

ROTHMAN, K.J.; GREENLAND, S.; LASH, T.L. Modern Epidemiology. 3a ed. Philadelphia: Lippincott Williams \& Wilkins, 2008.

VENÂNCIO, S.I. et al. Avaliação para a Melhoria da Qualidade da Estratégia Saúde da Família - AMQ: estudo de implantação no Estado de São Paulo. São Paulo: Instituto de Saúde, 2008. 


\section{Content validity of the Evaluation for Quality Improvement of the Family Health Strategy} This paper aims to analyze the content validity of selfassessment tools that make up the Evaluation for Quality Improvement (EQI) of the Family Health Strategy (FHS). It is a retrospective ecological study on secondary data available from 46 out 64 municipalities participating in the EQI program until December 2007 in Espírito Santo state, Brazil, where 241 family health teams were identified. The average percentage of "yes" responses was calculated from data provided by health teams for each quality standard component of the EQI. There was a nonlinearity between the implementation complexity of work processes suggested by the EQI from the list of quality standards on each quality level and self-assessment of the health teams of municipalities surveyed. The non-linear relation between the quality levels of EQI and complexity of implementation of work processes recommended by the standards of quality shows the need to rearrange the terms of self-assessment, establish new quality standards and to find a more consistent correlation between the difficulty of implementation of work processes in FHP and quality levels.

> Key words: primary health care, family health, quality of health care. Evaluation for Quality Improvement of the Family Health Strategy. 